\title{
SMAC2003: The Automatic Iteration of SMAC
}

\author{
Brian G. McHenry and Raymond R. McHenry \\ McHenry Consultants, Inc., Cary, NC
}

Copyright (@ 2003 SAE International

\begin{abstract}
In prior studies researchers have been interested in automating the process by which the Simulation Model of Automobile Collisions (SMAC) is used to reconstruct an accident. The SMAC program requires an initial approximation of the impact speeds and the positions and orientations at impact. And with a SMAC reconstruction you can sometimes get a reasonably close match and then spend many hours on iterative runs trying to match as best as possible the overall body of physical evidence.
\end{abstract}

The prior research on automation of SMAC (during the time period 1975-1980) was constrained by computer time and resources. Those research projects were performed on mainframe computers where all applications included charges for CPU time and memory resources. Today with gigahertz Pentium computers and unlimited memory, aside from the initial cost of the computer, the cost per SMAC run is virtually free and the time for a run is measured in seconds rather than minutes.

This paper describes an automatic iterative procedure which can quickly and efficiently iterate to a "best match" of the physical evidence with SMAC. Quantitative measures of the overall "fit" to the evidence, which guide the procedure, are discussed. Representative results from applications to experimental tests are presented.

\section{INTRODUCTION}

The process of reconstructing a motor vehicle collision involves collecting all available information about the interaction of the vehicles including vehicle trajectory information, damage information, vehicle specifications and scene information.

The trajectory information is gathered based upon the police measurements, photographs and scene evidence documentation (skid marks, gouges, etc.). To characterize the interaction of the vehicles the approximate location of the area of impact, the measured positions of rest and any skid and gouge marks should be memorialized. Technological advances in survey and measurement equipment have made equipment available to police and investigators which can quickly, efficiently and accurately memorialize vehicle accident scenes (e.g., Figure 1).

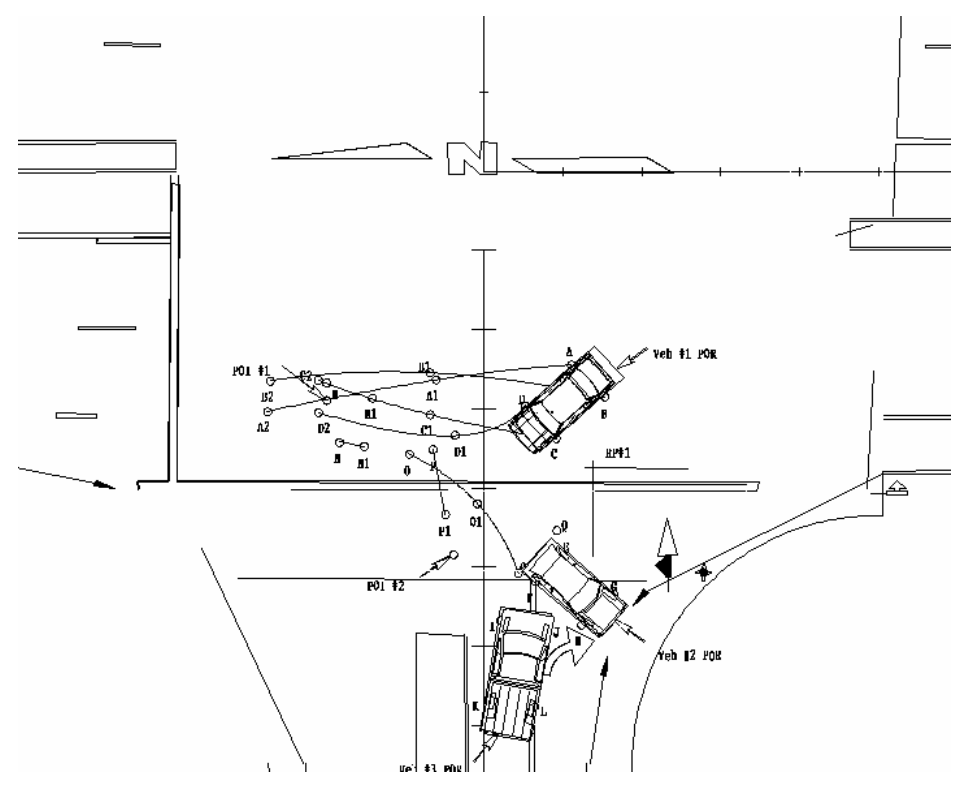

Figure 1 Sample scene diagram with tire mark
evidence measurements

The damage information includes measured dimensions of the damage locations and extents (e.g., Figure 2). The standard procedure by which damage is characterized is the Collision Deformation Classification $\left(C D C,[1]^{1}\right)$ and the Equidistant Crush Measurement (ECM, [2]).

Collecting vehicle specifications and scene information (roadway layout and topography) completes the required data to permit the performance of an accident reconstruction.

Collision reconstruction techniques are then applied to make a preliminary determination of the impact speeds and impact speed changes ( $\Delta \mathrm{V}$ 's). There are two basic reconstruction techniques utilized for performing a preliminary accident reconstruction: Trajectory analysis techniques and damage analysis techniques.

\footnotetext{
${ }^{1}$ Numbers in brackets [ ] are references at end of paper
} 

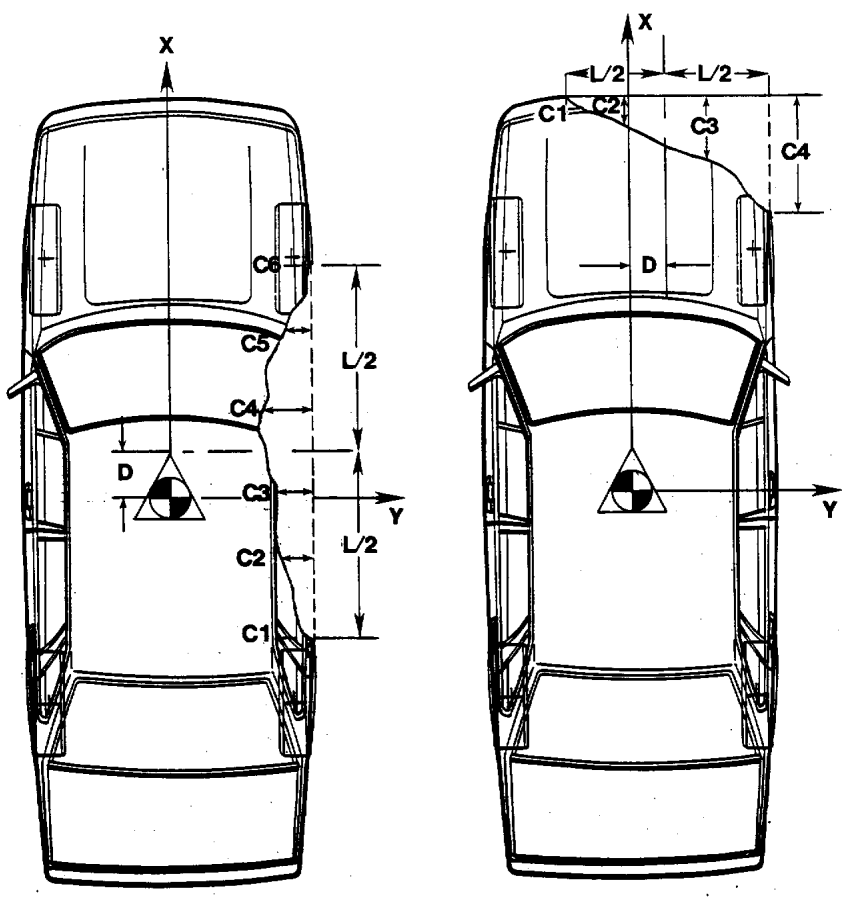

Figure 2 Sample damage dimensions

Trajectory analysis techniques are based on applications of the principles of the Conservation of Linear and Angular Momentum and they frequently include the simplifying assumptions of instantaneous exchange of momentum, no consideration for tire-to-ground "external" forces during the collision, and straight line travel from separation to rest [3, p84-85].

Damage analysis techniques are generally based upon the work of Emori [4], Campbell [5] and the CRASH damage algorithms [6]. These damage analysis algorithms are predominantly based on measurements from rigid, fixed barrier crash tests without consideration of restitution effects. As a result of the lack of distinction in the damage analysis techniques between stiffness and restitution effects, the "virtual" crush model can result in substantially different vehicles sharing nearly equal slopes and intercepts in CRASH type plots of the approach period speed-change as a function of residual crush. Such similarities can occur even though the actual exposure severity for a given residual crush may be significantly different [7].

In recognition of the generally simplistic nature of trajectory and damage analysis techniques, NHTSA sponsored research projects to develop computer programs that would achieve improved uniformity, as well as improvements in accuracy and detail, in the interpretation of physical evidence in highway accidents. One resulting computer program was the Simulation Model of Automobile Collisions (SMAC, [8]).

SMAC is a time-domain mathematical model in which the vehicles are represented by differential equations derived from Newtonian mechanics combined with empirical relationships for some components (e.g., crush properties, tires) that are solved for successive time increments by digital integration.

The SMAC computer model is an "open-form" of reconstruction procedure wherein the user specifies the dimensional, inertial, crush and tire properties of the vehicles, the initial speeds, angles and driver-control inputs. The program, through step-wise integration of the equations of motion, produces detailed time-histories of the vehicle trajectories including the collision responses. The user compares the SMAC-predicted trajectories and collision deformations with the physical evidence to determine the degree of correlation. Iterative runs can then be performed, varying initial speeds, heading angles, control inputs and damage effects until an acceptable match of the physical evidence is achieved.

As any SMAC user is aware, many iterations of the program may be required to go from an initial approximation to an acceptable match of the measured trajectory and damage targets. Throughout the iterative process, the impact speeds and speed change results may not change significantly. Also, what constitutes an acceptable match can vary widely among users. Sometimes the focus is on a detailed match of the positions of rest; sometimes the focus is on a match of damage locations and extents on the vehicles. There is currently no standardized measure of the correlation of SMAC results with the accident evidence.

Since the initial development of the SMAC program, there has existed a need to simplify the application process. The ultimate simplification would entail an automatic iteration procedure.

The working hypothesis of the presently described research on the automatic iteration of SMAC, as well as that of other simulation-type analytical approaches to accident reconstruction, is that a unique set of impact conditions is required to achieve an acceptable match of all of the documented evidence (both damage and trajectory). The use of quantitative measures of the overall "fit" to the documented evidence and applications to experimental crash tests provide a means of testing the hypothesis, as well as demonstrating reconstruction accuracy and convergence rates.

\section{PRIOR WORK ON AUTOMATIC ITERATION OF SMAC}

In 1975, Jones [9] presented a plan for automatic iteration of SMAC to make the SMAC program "user orientated" so that users can operate the program with "ease". The program modifications iterated only the vehicle speeds. The initial starting speeds of the vehicles for the SMAC runs were based on the results of the START2 routine [8, p88-96]. The SMAC predicted positions of rest were then compared with the measured positions of rest. A conclusion of the research was that "the results suggest that to obtain unique solutions for 
certain accident configurations it is insufficient to optimize on rest positions alone".

In 1980, Moffatt and Byrd [10] created a program entitled AUTOSMAC which automatically adjusted the starting conditions of the simulation in an attempt to match the target conditions. The target conditions which "may be used" included:

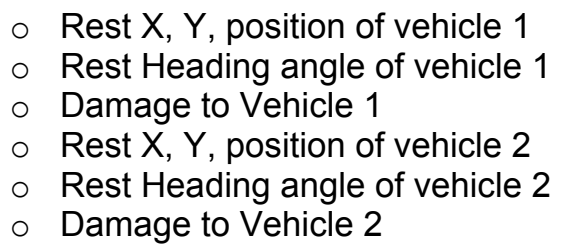

The variables which were optimized included the vehicle speeds, steering and braking. They found "the most difficult variables are steering and braking because collision outcomes such as rest positions relate in nonlinear ways to steering and braking". An optimization method was developed in view of the fact that "standard optimization procedures which make many trials of the system would be impractical because the SMAC program is costly to run". The authors found that only a few of the desired target conditions could be optimized due to limitations on time and budget.

These early research projects on the automatic iteration of SMAC were severely constrained by computer time and resources. The research was performed on mainframe computers where the cost of applications included charges for CPU time and memory resources. SMAC computer runs on mainframe computers were expensive, with "two or three iterations at a cost of $\$ 40$ \$60" [9]. From the early 1980's until the mid 90's, phenomenal advances in computer technology, particularly microprocessor technology [11], moved the mainstay of scientific computing from mainframes to mini-computers to personal computers. With personal computers there are no charges for CPU or memory usage.

In 1997, as part of research contained in the paper CRASH-97 [3], the authors implemented an update to the trajectory simulation routine of CRASH which included the automatic iteration of SMAC to simulate the trajectory of vehicles between separation and rest. The SMAC program was thereby used to refine and test the results of the CRASH program. The automatic iteration included adaptations of optimization techniques for error reduction and convergence in iterative solutions.

In 2001, with the advent of the Gigahertz+ Pentium 4 machine, the authors concluded that it was feasible and practical to extend the automatic iteration of SMAC development started with the $\mathrm{CRASH}-97$ research and to create an automatic iteration scheme for the complete SMAC program, including approach, collision and separation-to-rest phases.

\section{PROBLEM STATEMENT}

Manual iteration of SMAC is generally based on ad hoc comparisons of trajectory predictions with measured positions of rest and tire marks and of damage predictions with measurements. There exists a general need to create an objective, quantitative and nondimensional measure of the overall "fit" of the SMAC results to the documented evidence to supplement the existing graphical display outputs (e.g., Figure 3). Such a development is essential to the automated iteration procedure. The non-dimensional correlation "score" can serve to guide the user during either a manual or an automatic iterative process. Eventually, such a SMAC correlation score may be rigorously correlated with the reliability of the reconstruction results.

To create a quantitative measure of the overall fit, a function had to be created which would be minimized as the match of the physical evidence is improved. The following items of evidence have been used as components to represent a correlation factor score of the SMAC results:

\section{Trajectory measurements}

- The approximate positions and orientations of the vehicles at impact

- The measured positions and orientations of the vehicles at rest

- Distance POI to POR for each vehicle

- Azimuth angle POI to POR for each vehicle

- Direction of the System Momentum

\section{Damage measurements:}

\author{
- Damage width \\ Damage depth \\ Damage area \\ Centroid of the damage region \\ Clock direction of the approximate PDOF
}

\section{Table 1 Factors considered in the SMAC correlation comparison and score}

The input routines for SMAC were modified to include user input of the evidence target values for comparison with the SMAC predicted values. A routine was created to calculate differences between the measured targets and predicted values which are then combined and a resulting Correlation Factor "score" calculated. For damage measurement comparisons, an algorithm was developed in SMAC to scan the predicted damage tables and create 6-point Equidistant Crush Measurements based on the predicted damage tables, for use in the calculation of damage area, centroid, etc. (See Figure 4).

Once testing began it became apparent that some additional decisions had to be made as to what was the best manner in which to combine all the correlation calculations (Table 1) into a single function. Tests were 
performed on different combinations for the correlation factor score including addition of the absolute values of each and taking the square root of the sum of the squares of the individual errors.

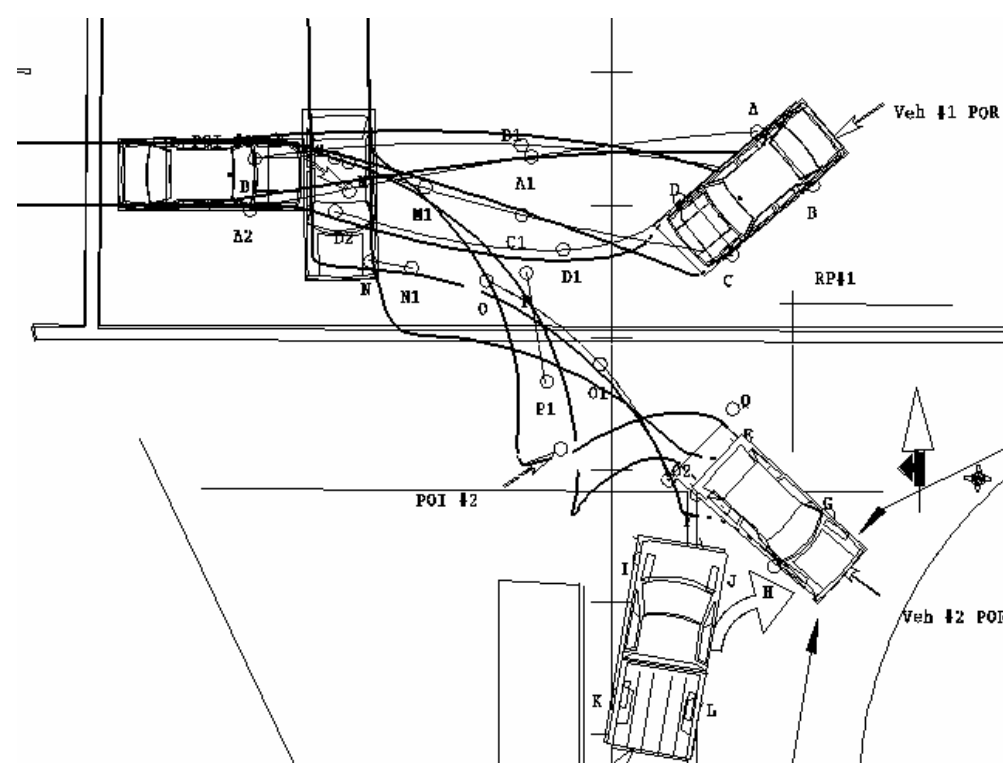

Figure 3 Sample SMAC graphics output with predicted vehicle trajectories on the measured scene evidence display

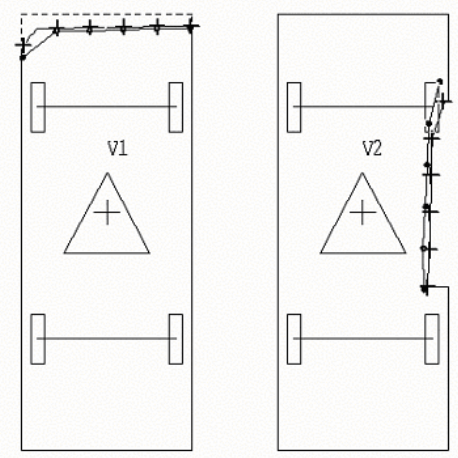

Figure 4 Sample SMAC predicted damage output including a SMAC generated 6-point damage profile

Many different optimization and error minimization routines were investigated [12-16]. A fundamental problem with the use of many of the investigated control algorithms was the inherent requirement that the functions must be continuous and/or linear. The collision and trajectories of vehicles can be highly non-linear events. Minor variations in starting conditions (i.e., speed, impact location) can produce major changes in the resulting rest positions $(\mathrm{X}, \mathrm{Y}, \mathrm{PSI})$ and discontinuities in the calculated error evaluation terms. For example, during decelerations of the linear and angular velocities, as a vehicle rotates while it travels from separation to rest, the vehicle may "shoot off" tangentially in what has been described as a "dog leg" type of trajectory at any time that the velocity vector aligns with the longitudinal axis. Traditional function minimization techniques which require the evaluation of some form of derivatives (e.g., Cramer's rule, Newton's method) or include the assumption of a linear function (Powell's method, Broyden's method) were found to fail in many instances where step changes were produced in the "function" by minor alterations of the variables. The final form of the function minimization routine is a customized routine roughly based upon an adaptation of the downhill simplex method of Nelder and Mead [17] and Press [15].

Weighting factors were tested and applied to the individual errors in the evidence match to help improve the efficiency of the iterative process. The weighting factors assist the iterative function by forcing primary focus on the most important errors to achieve a gross match of the directions of vehicle travel and areas of damage. Secondary items of evidence are the detailed positions and orientations at rest. At periodic intervals during the iterative process, the patterns of evidencematch errors are scanned to provide a basis for corresponding step adjustments in the inputs of the iterative process. The input adjustments are applied in automatic restarts which are aimed at an increased rate of convergence. Evaluations of the overall match of the documented evidence are based entirely on the unweighted measures of the individual errors.

\section{VALIDATION AND TESTING}

One of the problems associated with the development and refinement of any accident reconstruction technique is that of demonstrating correlation with full-scale tests. The RICSAC tests $[18,19,20]$ were specifically designed to serve as standards for such comparisons. Unfortunately, during review and utilization of the results (e.g., [21, 22, 23]), and particularly in prior studies which included evaluating the correlation of computer codes with RICSAC (e.g., [24-28]) there have been various levels of interpretation and acceptance of the measured results. As a part of many of the cited projects, questions have been raised as to the validity of some of the reported RICSAC test results.

Since there was no consensus on the interpretation of some of the results of the RICSAC tests, an intensive independent effort was applied by the authors toward achieving proper and generally acceptable interpretations of the RICSAC test data. The results are reported in RICSAC-97 [29]. That paper presented a detailed review and decipherment in useable form of the original 12 crash tests that were performed within the RICSAC program. In 2002, Brach and Smith [30] reanalyzed the RICSAC data from inertial coordinates and confirmed that the results of the RICSAC tests as interpreted in the RICSAC-97 research are appropriate and valid for comparing experimental data with predictions from accident reconstruction models. 


\section{STARTING VALUES FOR ITERATION}

The general validation procedure for SMAC to date has been to reconstruct full-scale tests starting with the known impact speeds and to then iterate the positions of impact and damage effects until a reasonable match of the positions of rest and damage was achieved. The creation of the presently reported automatic interaction procedure for SMAC (SMACITER) provides a unique opportunity for additional validation of SMAC by applications to test data which do not make any use of a prior knowledge of the initial speeds of impact.

In recognition of the original intent of the CRASH program, it was decided to use CRASH results as the starting point for the SMACITER iterative procedure. The original form of the CRASH $[6,31-33]$ computer program, which culminated in the CRASH3 version, was not intended to be a detailed, highly accurate reconstruction program. Rather, it was developed to serve as a simple pre-processor for the SMAC program. While the results of CRASH3 applications can be useful in providing approximate measures of accident severity for use in statistical studies, where the average error is most important, it has been demonstrated in validation studies to produce results which when compared to those of full-scale crash tests can include individual errors as great as $45 \%[33,3]$.

Although the possible error levels with CRASH are high it was decided to explore whether a CRASH type approximation technique would be adequate to create starting values for the SMACITER procedure. The standard inputs for CRASH, which include definition of the damage and trajectory accident evidence, are the needed information to start an iterative SMAC run (Table 2).

1. Approximations of impact positions and headings.

2. Measurements of rest positions and headings.

3. Approximations of wheel steer and drag of vehicles from separation position to rest.

4. Vehicle specifications (weights, dimensions, yaw moment of inertia, tire properties, etc.).

5. Damage measurements per Collision Deformation Classification (CDC, [1]) and the Equidistant Crush Measurement (ECM, [2]).

\section{Table 2: Minimum Information required for the CRASH program to be used as a starter for SMACITER}

\section{DEVELOPMENT PROCEDURE}

The iterative process consists of automatically adjusting the SMAC inputs to achieve reductions in the magnitude of the non-dimensional error function. Since the correlation factor "score" is based on the combination of a large number of individual measures of the evidence fit, it is possible that a "minimum" value may be only local. Therefore, to insure that the automatic iteration scheme will avoid local minimums, several restarts of the procedure with relatively large input adjustments are used to seek a global, or overall, minimum.

The iterative process makes use of simple logic with which the results of a series of successive adjustments in the individual variables such as the vehicle speeds, their positions and headings at the start of the approach, steer effects during and after the collision (a single fixed value), are used as a basis for the selection of a combination of adjustments. Following the combination of adjustments, the procedure is repeated.

In each iterative run of SMAC, the physical laws are fully applied without any mathematical shortcuts or user intervention. Thus, the achieved evidence match can be duplicated by a single SMAC run that uses the same inputs.

In the beginning stage of development, to simplify the development of the iterative procedure, SMAC runs which closely match the RICSAC tests were established as the initial targets for evidence matches. In this manner, the "evidence" was made to be completely compatible with the iterative reconstruction procedure.

During the spinout of a vehicle, an undamaged steering system will respond to side forces. The phenomenon was modeled with the HVOSM steer-degree of freedom model [34]. Tests were performed of the usefulness of incorporating either a steer degree-of-freedom option or a path follower option [35, 36], and/or optimizing by means of the steer tables alone to improve the rest position match when a vehicle's trajectory indicates the presence of steering system effects subsequent to the initial impact. The iteration scheme was modified to include optional single vehicle iterations which provide testing and refinement of the steering input approximations during the automatic restarts previously discussed.

\section{DISCUSSION OF RESULTS}

The following is a brief presentation and discussion of representative results obtained with the automatic iteration of SMAC. In each presented test the CRASH program was used to approximate the starting collision speeds for SMACITER, which was then used to refine the impact speeds and improve the match of the evidence.

In the first two tests presented, RICSAC Test\#11 and RICSAC Test \#4, the automatic iteration procedure uses 
as "evidence" SMAC generated results. The SMAC generated results were manual reconstructions of the RICSAC full-scale tests. The use of mathematically correct SMAC results was used to initially test and refine the automatic iteration procedure prior to application of the procedure to the actual full scale tests results.

In the next two tests presented, RICSAC Tes\#8A and RICSAC Test\#8, the automatic iteration procedure is used to demonstrate first iterating to a match of SMAC created "evidence" (Test\#8A) and then secondly used to demonstrate iteration to the actual RICSAC full-scale test results (Test\#8). Comparison of the results of Test\#8A and Test\#8 reveal that although the damage evidence is not exactly matched in the full-scale test comparison (Test\#8), the iterative procedure still improved the overall correlation of the results with the evidence.

The last test presented is for RICSAC Test\#2 and is a demonstration of iteration from the initial CRASH computer speeds to a match of the RICSAC full-scale test results.

\section{DETAILED DISCUSSION OF THE RESULTS}

RICSAC Test\#11: In Figure 5 and Figure 6, SMACgenerated positions and headings at rest and damage profiles, corresponding to the vehicle properties and impact conditions of RICSAC Test\#11, are displayed as the "target" evidence. Also in Figure $\mathbf{5}$ and Figure $\mathbf{6}$, the results of the initial run of SMAC, which utilized CRASHbased speed estimates, are displayed.

In Figure 7 and Figure 8, results of SMACITER are displayed subsequent to approximately 180 iterative adjustments of speed, impact positions and steer effects. Note that the impact speed errors in Figure 7 have been reduced by the iterative procedure to near $0.0 \%$.

The evidence match "scores" (i.e., deviations from a perfect match) are displayed as functions of the number of iterative runs in Figure 9. Note that the display includes the total correlation "score" as well as the components corresponding to the trajectory match and the damage match.

RICSAC TEST\#4: In Figure 10 and Figure 11, SMACgenerated positions and headings at rest and damage profiles, corresponding to the vehicle properties and impact conditions of RICSAC test \#4, are displayed as "target" evidence.

In Figure 10 and Figure 11, the results of the initial run of SMAC, which utilized CRASH-based speed estimates, are displayed. In Figure 12 and Figure 13, results of SMACITER are displayed subsequent to approximately 140 iterative adjustments of speed, impact positions and steer. Note that the impact speed error for Vehicle\#1 in Figure $\mathbf{1 2}$ have been reduced by the iterative procedure to approx. $2.0 \%$.
The evidence match "scores" (i.e., deviations from a perfect match) are displayed as functions of the number of iterative runs in Figure 14. The display includes the total correlation "score" as well as the components corresponding to the trajectory match and the damage match. Note that during the iterative procedure, the highly nonlinear behavior of the reconstruction is demonstrated by the erratic changes of the trajectory results. Damage to the rear of Vehicle\#1 produced drag on the rear of Vehicle \#1 and for certain offset configurations and/or speeds a spinout of the vehicle would occur. In the actual test the vehicle did not spin out and therefore when a spinout occurred a dramatic increase in the correlation score also occurred.

RICSAC Test\#8A: In Figure 15 and Figure 16, SMACgenerated positions and headings at rest and damage profiles, corresponding to the vehicle properties and impact conditions of RICSAC test \#8, are displayed as "target" evidence.

Also in Figure 15 and Figure 16, the results of the initial run of SMAC, which utilized CRASH-based speed estimates, are displayed. In Figure $\mathbf{1 7}$ and Figure 18, results of SMACITER are displayed subsequent to approximately 140 iterative adjustments of speed, impact positions and steer. Note that the impact speed errors in Figure $\mathbf{1 9}$ have been reduced by the iterative procedure to near $0.0 \%$.

The evidence match "scores" (i.e., deviations from a perfect match) are displayed as functions of the number of iterative runs in Figure 19. The display includes the total correlation "score" as well as the components corresponding to the trajectory match and the damage match.

RICSAC Test\#8: In Figure 20 and Figure 21, positions and headings at rest and damage profiles, corresponding to reported results of RICSAC test \#8, are displayed.

In Figure $\mathbf{2 0}$ and Figure 21, the results of the initial run of SMAC, which utilized CRASH-based speed estimates, are displayed. In Figure 22 and Figure 23, results of SMACITER are displayed subsequent to approximately 150 iterative adjustments of speed, impact positions and steer. Note that the impact speed errors in Figure $\mathbf{2 2}$ have been reduced by the iterative procedure to less than $10.0 \%$.

The evidence match "scores" (i.e., deviations from a perfect match) are displayed as functions of the number of iterative runs in Figure 24. The display includes the total correlation "score" as well as the components corresponding to the trajectory match and the damage match.

RICSAC Test\#2: In Figure 25 and Figure 26, positions and headings at rest and damage profiles, corresponding to reported results of RICSAC test \#2, are displayed. Also in Figure 25 and Figure 26, the results 
of the initial run of SMAC, which utilized CRASH-based speed estimates, are displayed.

In Figure $\mathbf{2 7}$ and Figure 28, results of SMACITER are displayed subsequent to approximately 140 iterative adjustments of speed, impact positions and steer effects. Note that the impact speed errors in Figure 29 have been reduced by the iterative procedure to approximately $1.0 \%$.

The evidence match "scores" (i.e., deviation from a perfect match) are displayed as functions of the number of iterative runs in Figure 29. The display includes the total correlation "score" as well as the components corresponding to the trajectory match and the damage match.

\section{DISCUSSION AND CONCLUSIONS}

The utilization of an automatic iteration of SMAC, or of any particular accident reconstruction technique, should not detract from the duty of the reconstructionist to perform a careful and detailed investigation and analysis of an accident. The reported research is directed towards developing an additional tool which should be used in conjunction with other techniques to perform the most accurate reconstruction that is possible for the data collected and the time available.

The reported results demonstrate that the SMACITER program successfully converges toward evidence matches in a variety of impact configurations. In initial applications wherein SMAC generated "evidence' was used the errors in impact speeds generally run less than approximately $\pm 2 \%$. With measured evidence from fullscale tests, wherein the deviations from a perfect evidence match run larger, the errors in impact speeds have been found to run less than approximately $\pm 10 \%$.

On the basis of the development effort to date, the following conclusions can be drawn:

1. The feasibility of an automated procedure for achieving a "best match" of measured evidence, starting with user responses to a CRASH-based series of questions, has been established. Small deviations from a perfect "match" are, of course, imposed by the existing limitations of the SMAC computer program and by any inaccuracies in the reported evidence.

2. The magnitude of the unweighted "score" of the achieved evidence match provides a general indication of (a) the quality of the measured evidence and (b) the reliability of the reconstruction results. Correlation of the "score" with measures of the reliability of speed results is considered to be a realistic future objective.

3. For efficiency of convergence, intermittent running adjustments in the weighting factors, controlled by the distribution of errors, appear to constitute an effective means of increasing the rate of convergence. Evaluations of the overall match of the documented evidence are, of course, based entirely on the unweighted measures of the individual errors.

4. The original purpose for the CRASH program was to give the SMAC user a running start for the iterative procedure by providing approximate starting speed results. The presently reported research adopts and advances the concept by using CRASH as a starting point and automating the sometimes tedious task of the SMAC iteration procedure.

5. The use of SMACITER does not require adoption of any new theories, assumptions, or techniques. The end product of a SMACITER application is a standard set of inputs for the SMAC program which will produce a "best fit" to the overall body of evidence. The manner in which the input data set for a "best fit" is obtained has no effect on the validity of the SMAC reconstruction results.

\section{FUTURE PLANS}

There are many areas of research which can benefit from the availability of an objective automatic iterative procedure for SMAC. The procedure is currently set up to permit testing of the relative sensitivities of individual variables. Many variables, such as the intervehicle friction coefficient, impulsive constraints on relative motion, and assumptions for crush stiffnesses, etc. can become better defined and understood and the relative importance of various assumptions can be evaluated by means of applications of SMACITER.

Comparison of SMACITER with all available full-scale crash tests will be compiled and reported in a future publication.

In those cases where tire marks are sufficiently documented, the existing graphics display of the "best fit" reconstruction will be supplemented by quantitative measures that compare the predicted and measured tire marks.

\section{CONTACT INFORMATION}

Questions or comments on the paper are welcomed and can be addressed to the authors by:

E-mail: mchenry@mchenrysoftware.com

Postal Service Mail:

McHenry Consultants, Inc.

103 Brady Court

Suite 200

Cary, NC 27511 USA

WWW: www.mchenrysoftware.com 


\section{REFERENCES}

1. SAE Standard J224 MAR80

2. SAE Standard J2433

3. McHenry, B.G., McHenry, R.R., "CRASH-97 Refinement of the Trajectory Solution Procedure", SAE Paper 97-0949

4. Emori, R.I., "Analytical Approach to Automobile Collisions", SAE Paper 68-0016

5. Campbell, K.L., "Energy Basis for Collision Severity", SAE Paper 74-0565

6. McHenry, R.R., "The CRASH Program - A simplified Collision Reconstruction Program", Proceedings of the Motor Vehicle Collision Investigation Symposium, Calspan, 1975

7. McHenry, R.R., McHenry, B.G., "Effects of Restitution in the Application of Crush Coefficients", SAE Paper 97-0960

8. McHenry, Raymond R., "Development of a Computer Program to Aid the Investigation of Highway Accidents", Calspan Report VJ-2979-V-1, NTIS PB \#208537

9. Jones, lan. S., "Automated Accident Reconstruction", SAE paper 750894

10. Moffatt, C.A., Byrd, J. Jr. :A Computer Model to Operate the SMAC Program Automatically, Highway Collision Reconstruction, Winter ASME Meeting 1980

11. http://www.intel.com/labs

12. Hostetter, G.H., Santina, M.S., D'Carpio-Montalvo, P. Analytical Numerical and Computational Methods for Science and Engineering, Prentice Hall, Englewood Cliffs, NJ 1991, ISBN 0-13-026055-X

13. Forsythe, G.E., Malcolm, M.A., Moler, Computer Methods for Mathematical Computations, C.B.,Prentice-Hall. Inc. Englewood Cliffs, NJ 1977, ISBN 0-13-165332-6

14. Etter, D.M., Fortran 77 with Numerical Methods for Engineers and Scientists, Benjamin/Cummings Publishing Company, Inc., 1994, ISBN 0-8053-17708

15. Press, W.H., Teukolsky, S.A.,Vetterling, W.T., Flannery, B.P., Numerical Recipes in Fortran. The Art of Scientific Computing ,Second Edition, Cambridge University Press, 1992 ISBN-0 521 $43064 X$

16. http://www.netlib.org/

17. Nelder, J.A., Mead, R. 1965 Computer Journal, vol 7, pp 308-313

18. McHenry, R. R., Lynch, J. P., Segal, D. J., "Research Input for Computer Simulation of Automobile Collisions", Calspan Report ZQ-6057-V1, Contract DOT-HS-7-01511, Jun 1977

19. Shoemaker, N. E., "Research Input for Computer Simulation of Automobile Collisions - Staged Collisions, Vol. II \& Vol. III", Calspan Report ZQ6057-V-4 \& V-5, Contract DOT-HS-7-01511, December 1978
20. Jones, I. S., Baum, A. S., "Research Input for Computer Simulation of Automobile Collisions Volume IV - Staged Collision Reconstructions", Calspan Report ZQ-6057-V-6, Contract DOT-HS-701511, December 1978

21. Smith, R.A., Noga, J.T., "Examples of Staged Collisions in Accident Reconstruction", presented at ASME Winter Meeting, Nov 1980 and contained in ASME publication "Highway Collision Reconstruction"

22. Brach, R. M., "Impact Analysis of Two-Vehicle Collisions", SAE Paper 83-0468

23. Bundorf, R.T., "Analysis and Calculation of Delta-V from Crash Test Data", SAE Paper 96-0899

24. Day, T.D., Hargens, R.L., "Further Validation of EDCRASH Using the RICSAC Staged Collisions", SAE Paper 89-0740

25. Woolley, R.L., "The "IMPAC" Computer Program for Accident Reconstruction", SAE Paper 85-0254

26. Wooley,R.L., Warner, C.Y., Tagg,M.D., "Inaccuracies in the CRASH3 Program", SAE Paper 85-0255

27. Cliff,E.C., Montgomery, D.T., "Validation of PCCRASH - A Momentum Based Accident Reconstruction Program", SAE Paper 96-0885

28. Day, T.D., Hargens, R.L., "Further Validation of EDSMAC Using the RICSAC Staged Collisions", SAE Paper No. 90-0102

29. McHenry, B.G., McHenry, R.R., "RICSAC-97 - A Reevaluation of the Reference Set of Full Scale Crash Tests", 97-0961

30. Brach, R.M., Smith, R.A., "Re-Analysis of the RICSAC Car Crash Accelerometer Data", SAE Paper 2002-01-1305

31. McHenry, R.R. Lynch, J.P., "User's Manual for the Crash Computer Program" Calspan Report No. ZQ-5708-V-3, Contract No. DOT-HS-5-01124, Jan 1976

32. NHTSA,"CRASH3 User's Guide and Technical Manual", Revised Edition, National Highway Traffic Safety Administration, DOT-HS-805732, April 1982

33. Tsongos, N.G., "CRASH3 Technical Manual", U.S. DOT, NHTSA, NCSS, Accident Investigation Division, July 1986

34. McHenry, R., R., DeLeys, N.J., "Vehicle Dynamics in Single Vehicle Accidents - Validation and Extensions of a Computer Simulation", Calspan Report VJ-2251-V-3, Dec 1968,

35. Glennon, J.C., McHenry, B.G., Neuman, T.R., "HVOSM studies of Highway Cross Slope Design", Contract DOT-FH-11-9575, NTIS PB 33191619; May 1982

36. McHenry, B.G., "Simulation Models of Vehicle Dynamics", Beyond the Green Book, TRB Circular, April 1992 


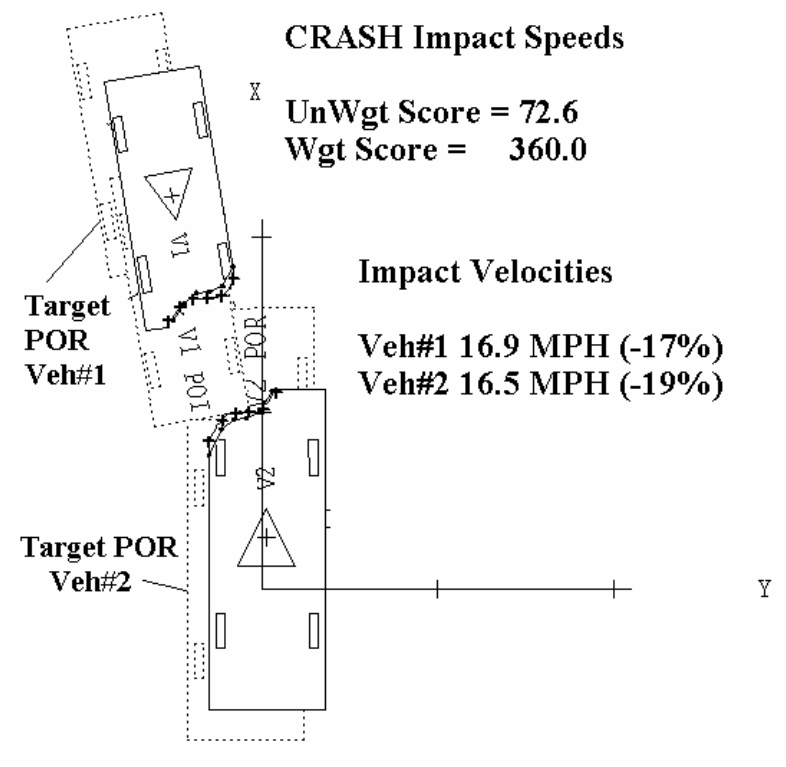

Figure 5 RICSAC Test\#11 starting point for SMACITER using CRASH impact velocities

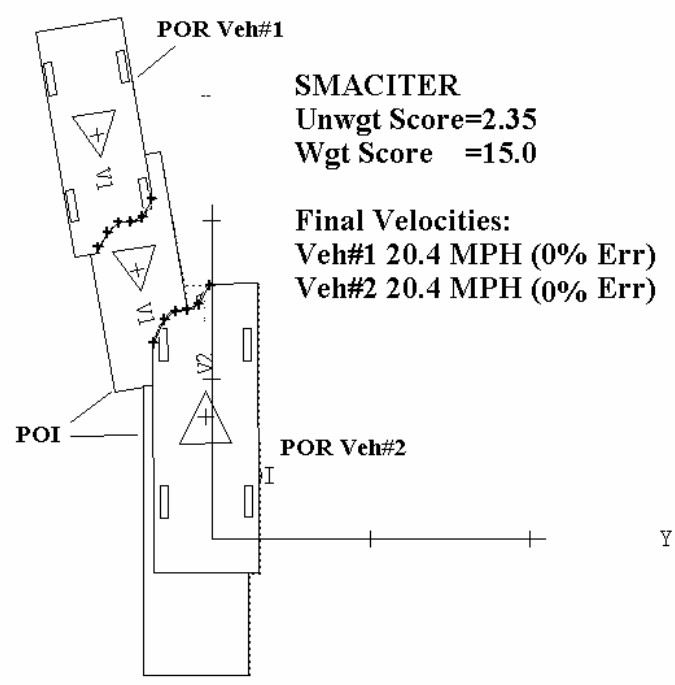

Figure 7 RICSAC Test\#11 Final Results

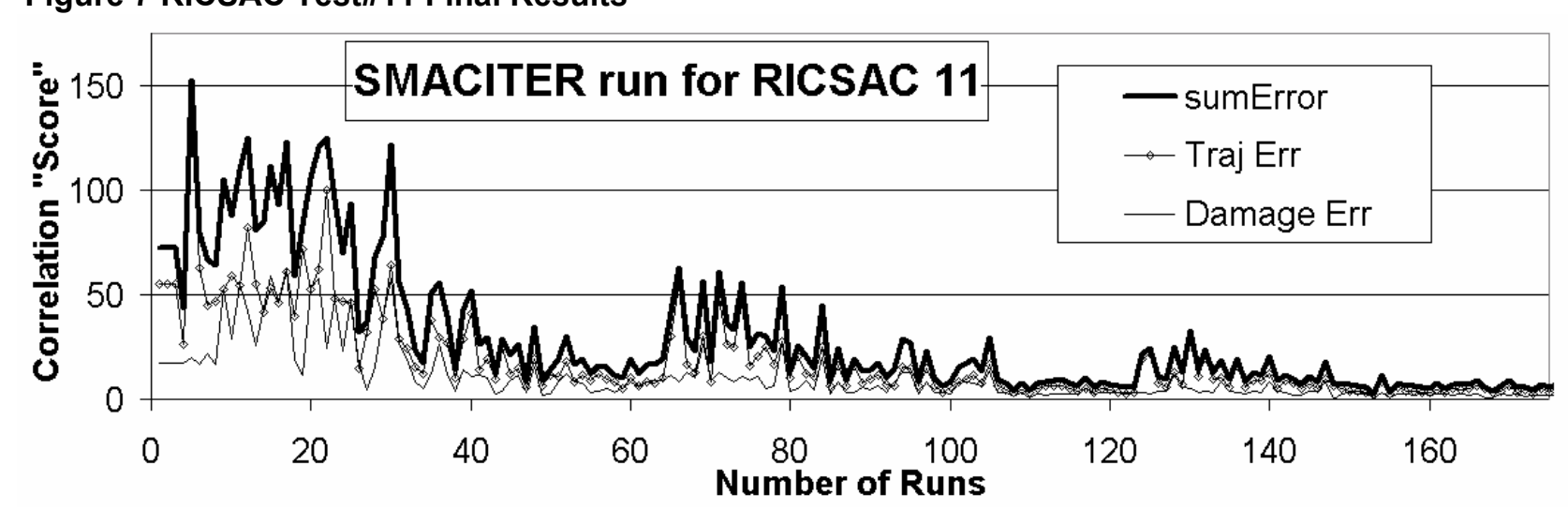

Figure 9 RICSAC Test\#11, SMACITER correlation "score" variation during iterative process
Figure 6 RICSAC Test\#11 starting SMAC Damage using CRASH Impact Velocities
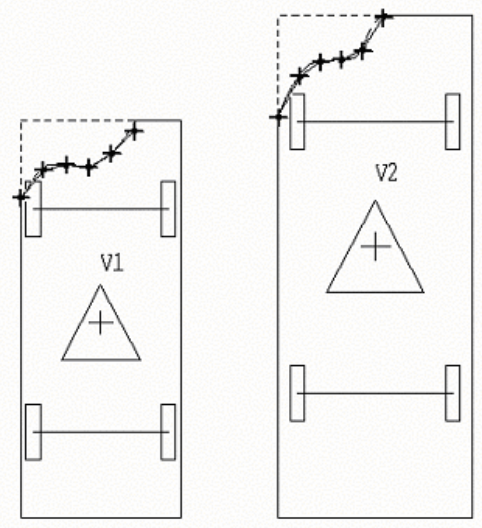

Figure 8 RICSAC test\#11 Final Damage Results 


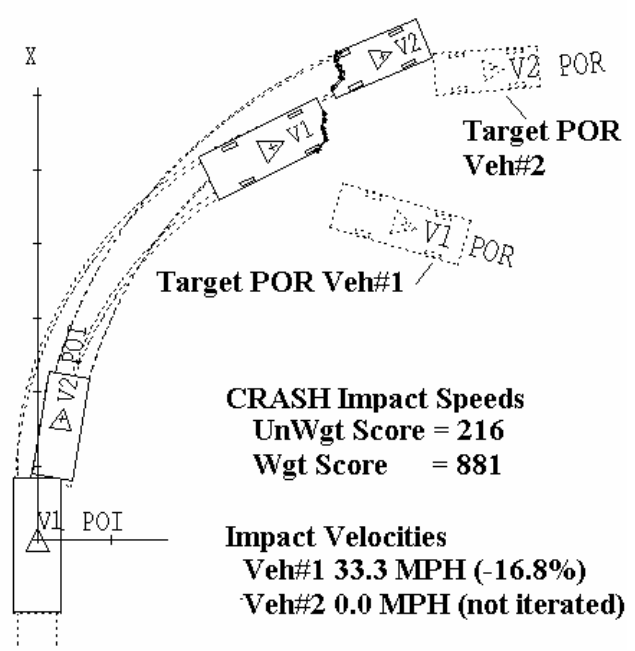

Figure 10 RICSAC Test\#4 starting point for SMACITER, using CRASH impact velocities

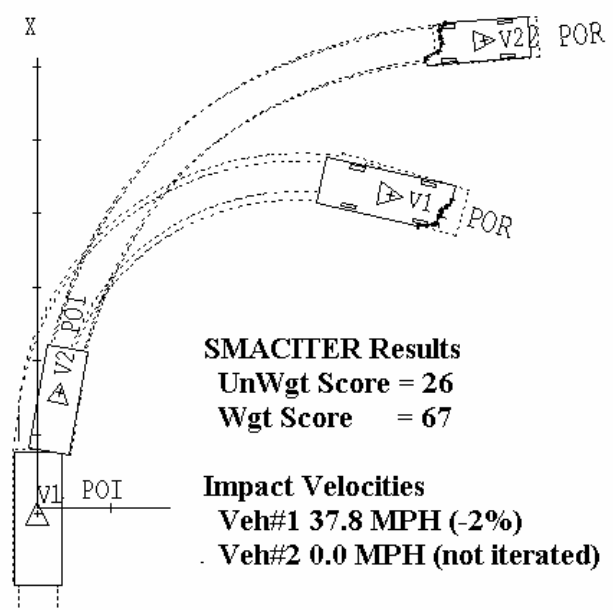

Figure 12 RICSAC Test\#4 Final Results

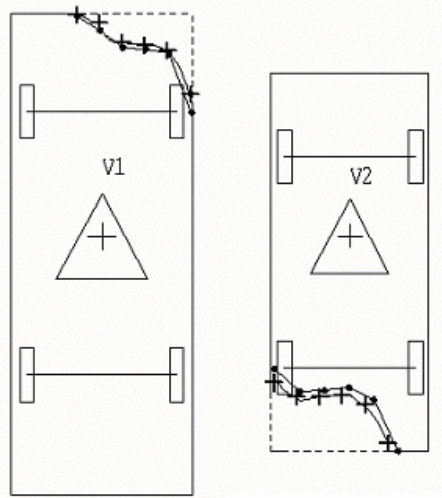

Figure 11 RICSAC Test\#4 starting SMAC Damage using CRASH Impact Velocities
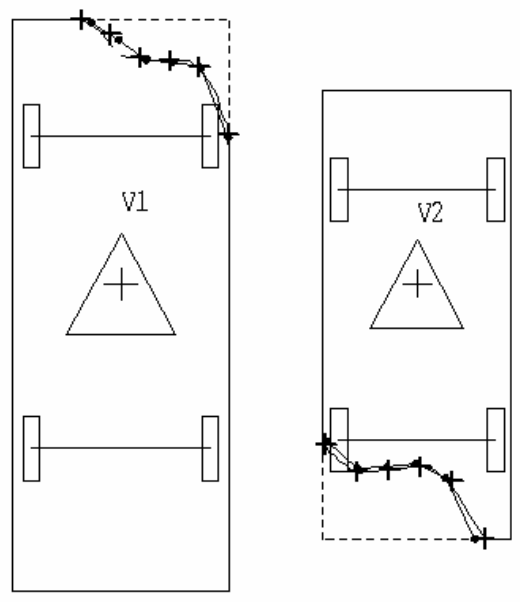

Figure 13 RICSAC Test\#4 final damage results

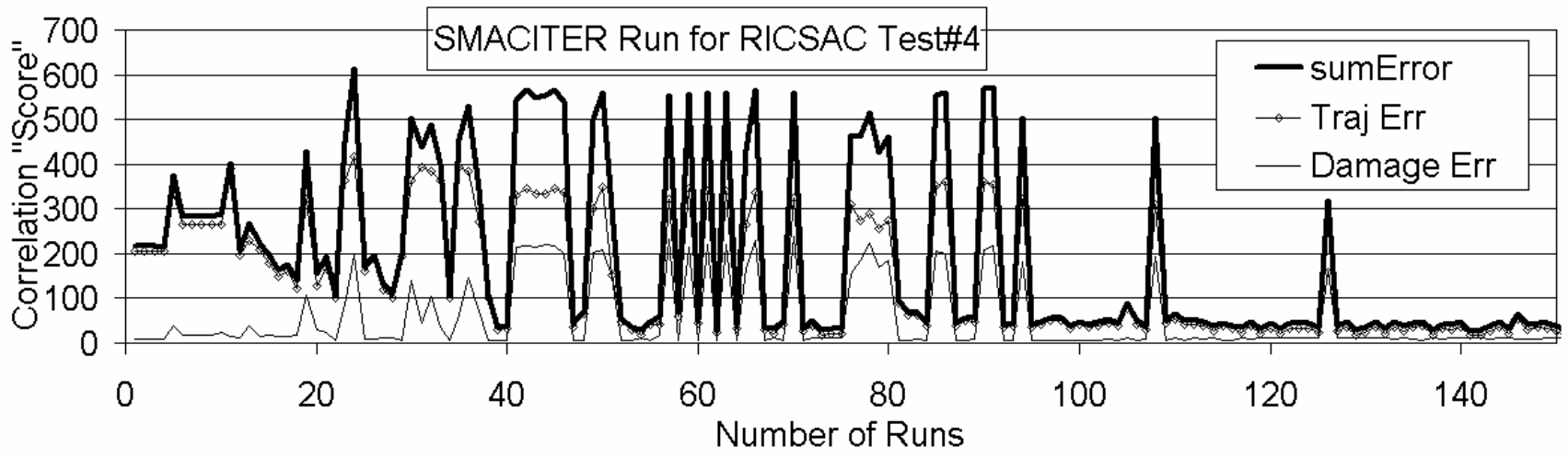

Figure 14 RICSAC Test\#4, SMACITER correlation "score" during the iterative process 


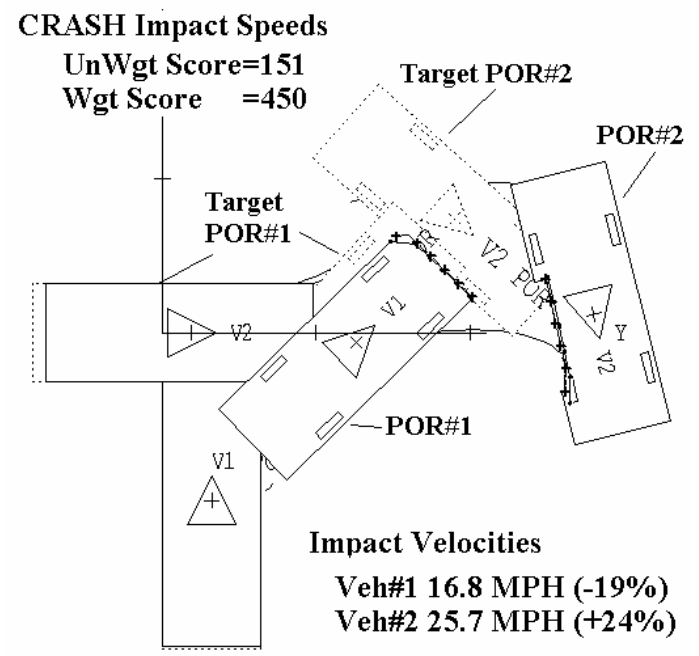

Figure 15 RICSAC Test\#8A starting point for SMACITER, using CRASH impact velocities

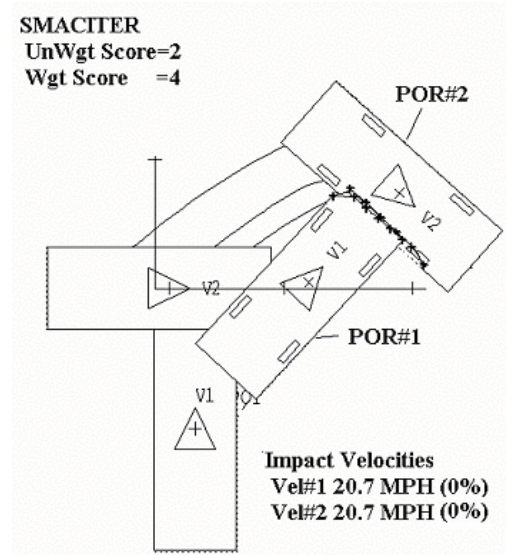

Figure 17 RICSAC Test\#8A final results of SMACITER

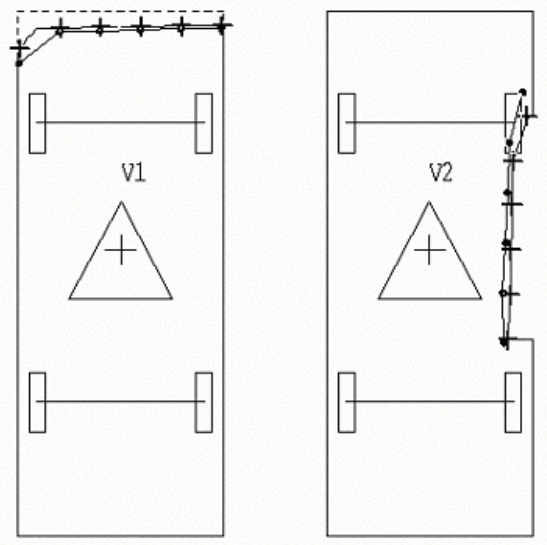

Figure 16 RICSAC Test\#8A starting SMAC Damage using CRASH Impact Velocities
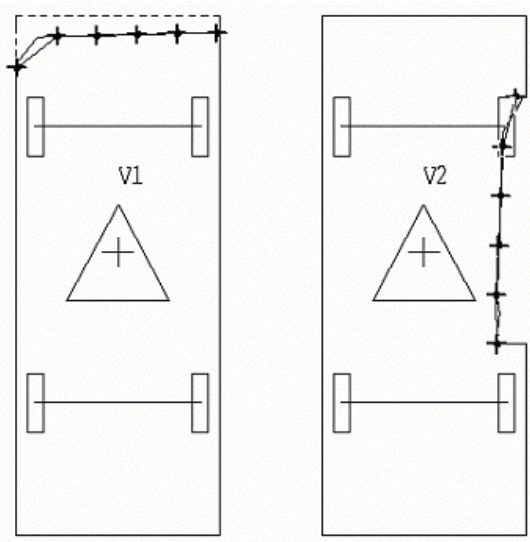

Figure 18 RICSAC Test\#8A final damage results

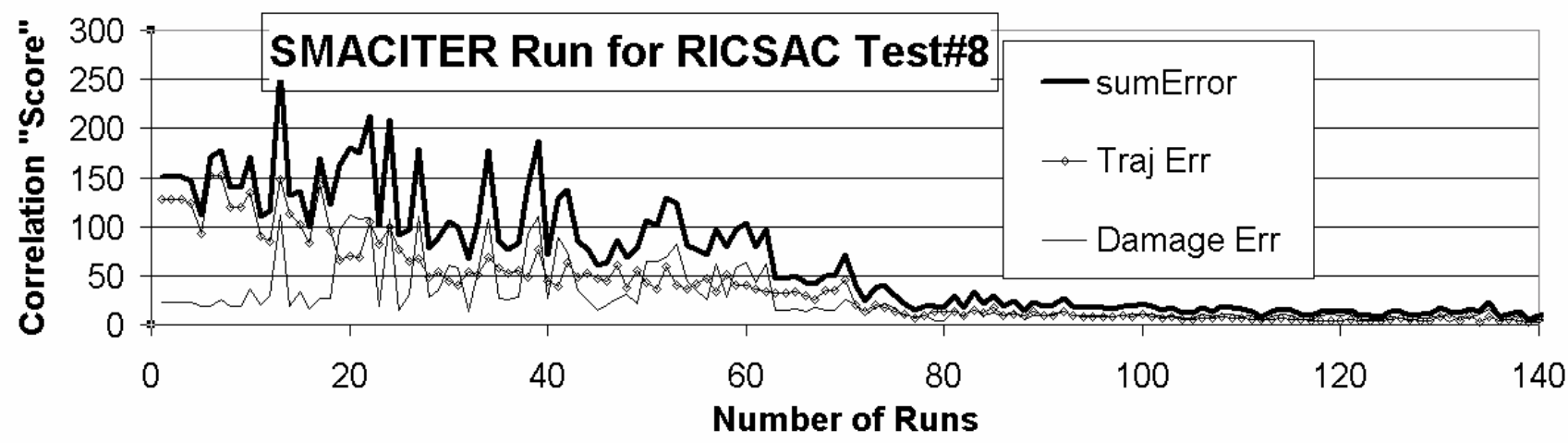

Figure 19 RICSAC Test\#8A, SMACITER correlation "score" during the iterative process 


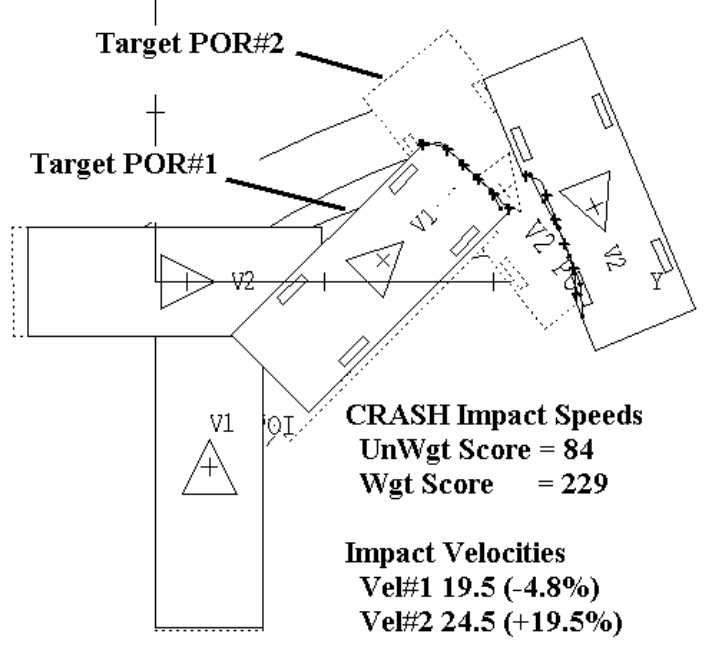

Figure 20 RICSAC Test\#8 starting point for SMACITER, using CRASH impact velocities

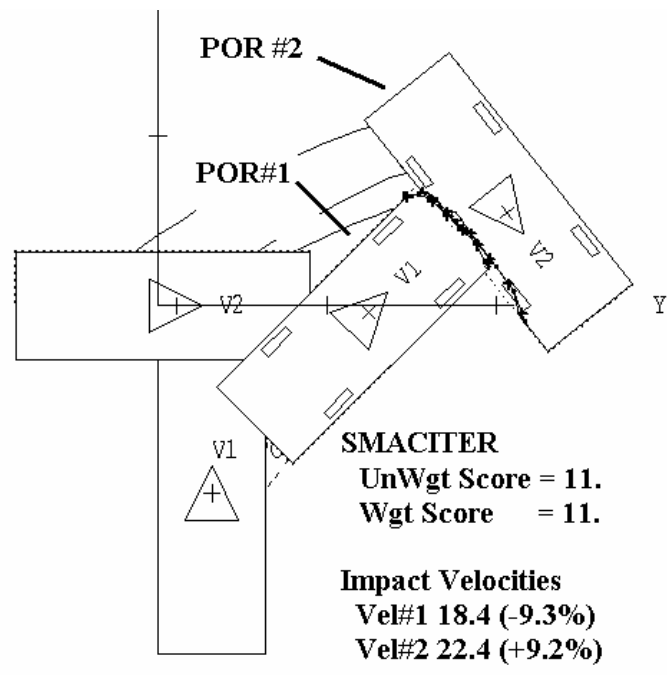

Figure 22 RICSAC Test\#8 final results of SMACITER
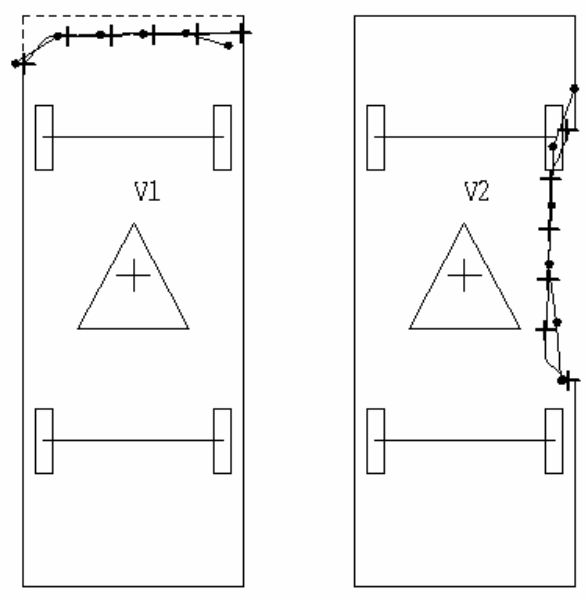

Figure 21 RICSAC Test\#8 starting SMAC damage using CRASH impact velocities
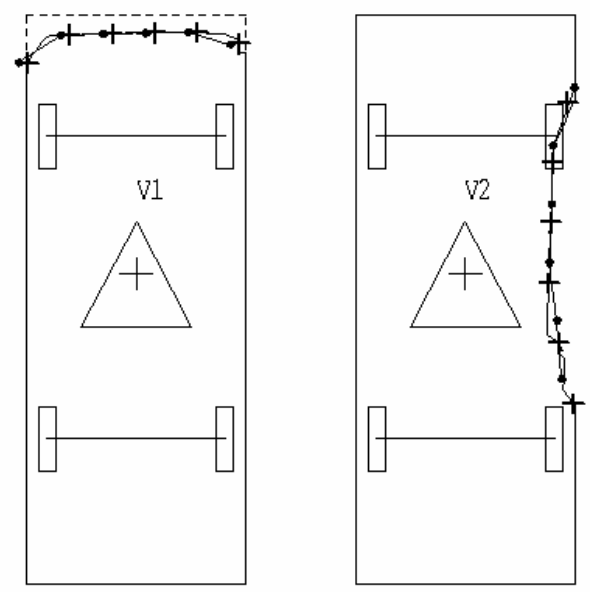

Figure 23 RICSAC Test\#8 final damage results

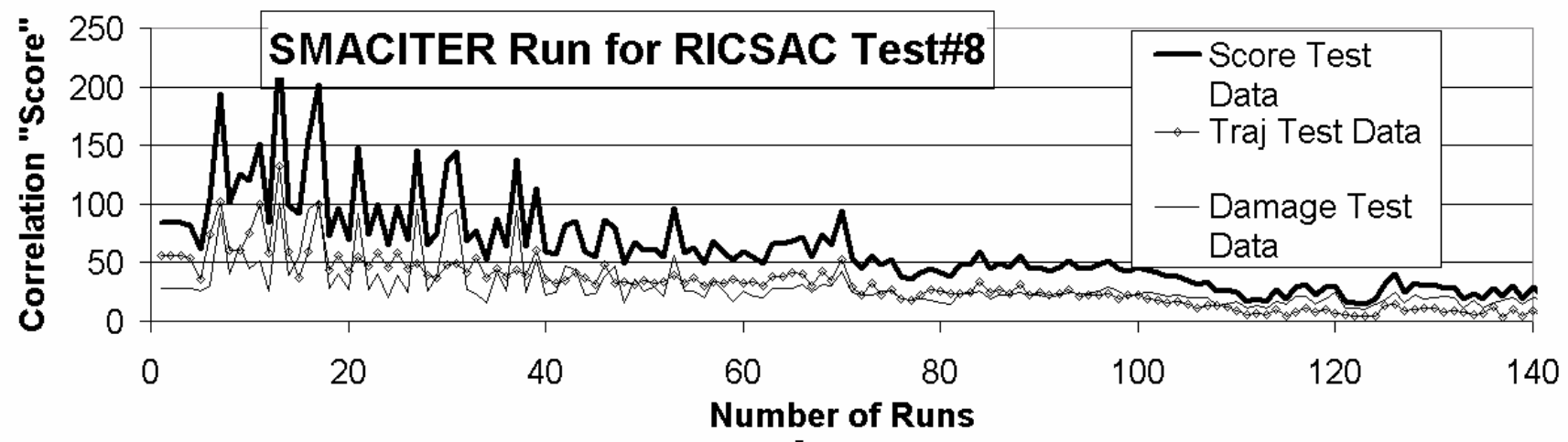

Figure 24 RICSAC Test\#8, SMACITER Correlation "score" during the iterative process 


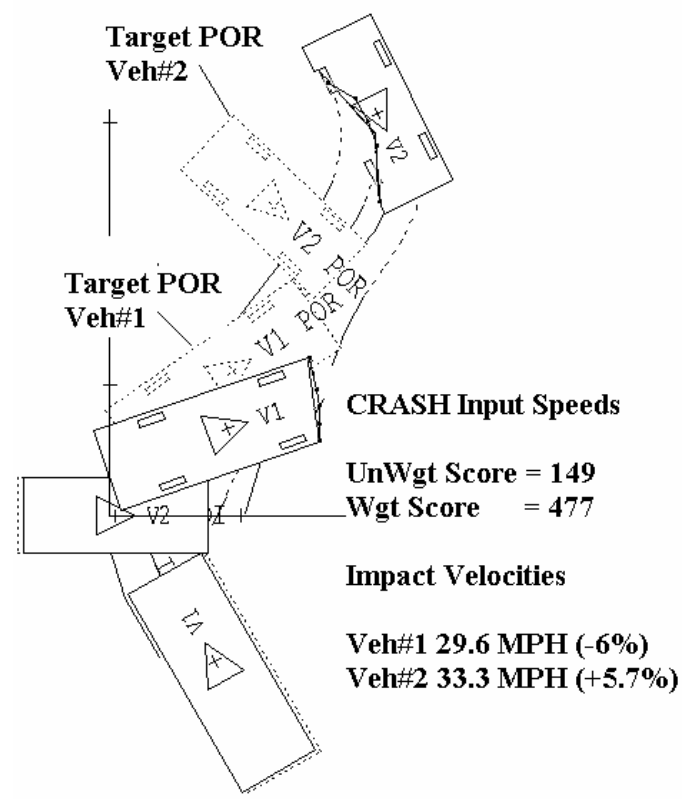

Figure 25 RICSAC Test\#2 starting point using CRASH impact velocities

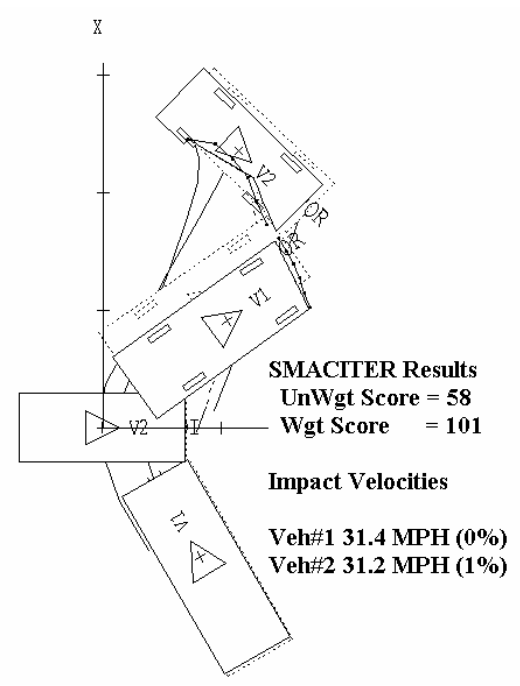

Figure 27 RICSAC Test\#2 final results or SMACITER

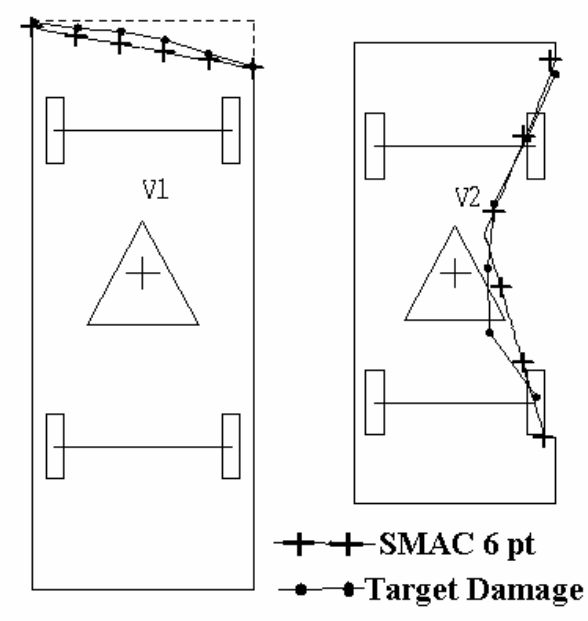

Figure 26 RICSAC Test\#2 starting SMAC damage using CRASH impact velocities

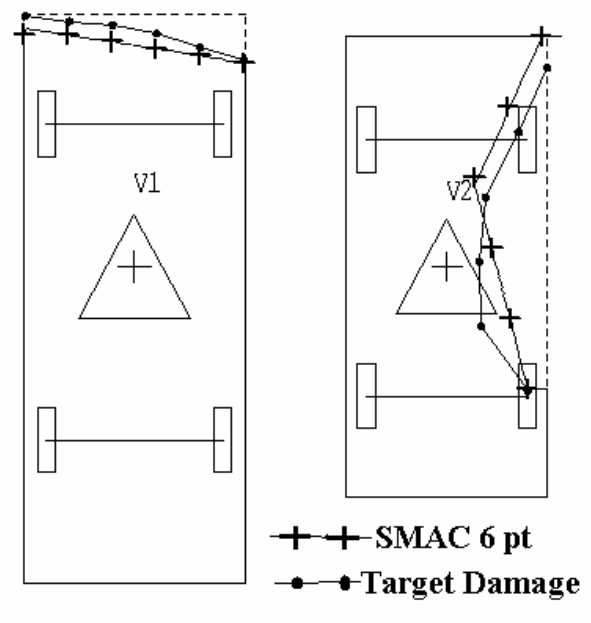

Figure 28 RICSAC Test\#2 final damage results

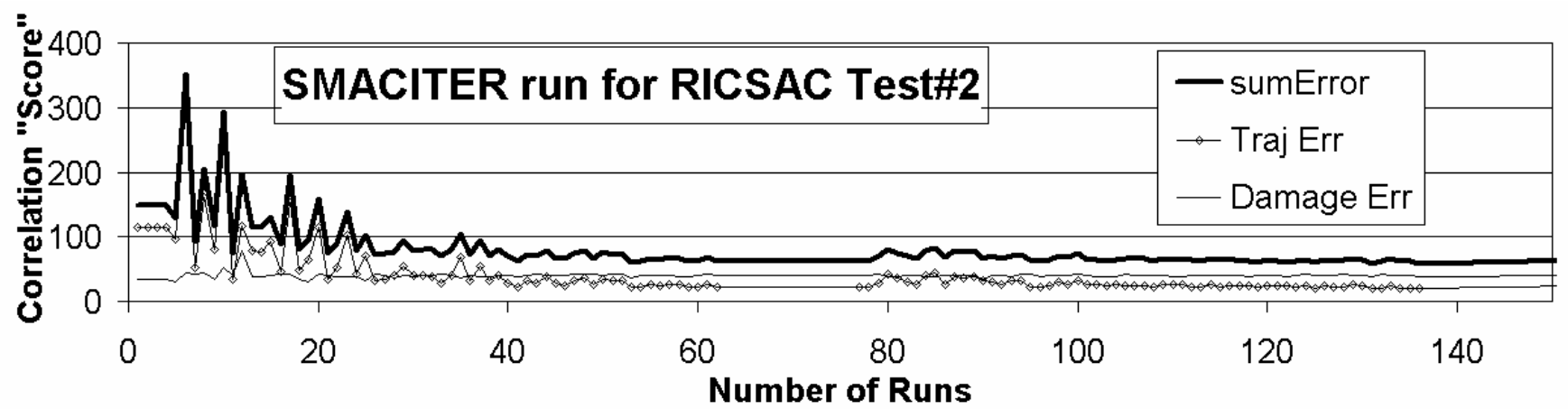

Figure 29 RICSAC Test\#2, SMACITER correlation "score" variation during iterative process 